\title{
Family of Functional Inequalities for the Uniform Measure
}

\author{
Khalid Boutahir ${ }^{1}$, Ali Hafidi ${ }^{2, *}$ \\ ${ }^{1}$ Département de Mathématiques \& Informatique, Université My Ismail, B. P. 11201 Zitoune, Meknès, MAROC \\ ${ }^{2}$ Faculté des Sciences et Techniques, B.P.509, Boutalamine Errachidia, MAROC \\ *Corresponding author: hafidiali28@gmail.com
}

\begin{abstract}
We consider on the interval $[-1,1]$ the heat semigroup $\left(P_{t}\right)_{t \geq 0}$ generated by the Legendre operator $L:=\left(1-x^{2}\right) \frac{d^{2}}{d x^{2}}-2 x \frac{d}{d x}$ acting on the Hilbert space $\mathbb{L}^{2}([-1,1], \mu)$ with respect to the uniform measure $\mu(d x):=\frac{d x}{2}$. By means of a simple method involving some semigroup techniques, we describe a large family of optimal integral inequalities with the Poincaré and logarithmic Sobolev inequalities as particular cases.
\end{abstract}

Keywords: heat semigroup, legendre operator, spectral gap, poincaré inequality, sobolev inequality, logarithmic sobolev inequality, $\varphi$-entropy inequality

Cite This Article: Khalid Boutahir, and Ali Hafidi, "Family of Functional Inequalities for the Uniform Measure.” Journal of Mathematical Sciences and Applications, vol. 5, no. 1 (2017): 19-23. doi: 10.12691/jmsa-5-1-3.

\section{Introduction}

Gross’ logarithmic Sobolev inequality [2] states that for all smooth functions $\mathrm{f}$ on $\mathbb{R}^{d}$

$$
\begin{aligned}
& \int_{\mathbb{R}^{d}} f^{2} \log f^{2} d \gamma_{d}-\left(\int_{\mathbb{R}^{d}} f^{2} d \gamma_{d}\right) \log \left(\int_{\mathbb{R}^{d}} f d \gamma_{d}\right) \\
& \leq 2\left(\int_{\mathbb{R}^{d}}|\nabla f|^{2} d \gamma_{d}\right),
\end{aligned}
$$

white $d \gamma_{d}$ denote the normalized Gaussian measure on $\mathbb{R}^{d}: d \gamma_{d}(x)=\left(\sqrt{2 \pi}^{-d}\right) \exp \left(-\|x\|^{2} / 2\right)$. In this Gaussian context, the Poincaré inequality(spectral gap inequality) is given by:

$$
\int_{\mathbb{R}^{d}} f^{2} d \gamma_{d}-\left(\int_{\mathbb{R}^{d}} f d \gamma_{d}\right)^{2} \leq \int_{\mathbb{R}^{d}}|\nabla f|^{2} d \gamma_{d} .
$$

In 1989, W.Bekner [2] derived a family of generalized Poincaré inequalities that yield a sharp interpolation between Poincaré inequality and logarithmic Sobolev inequality:

$$
\begin{aligned}
& \int_{\mathbb{R}^{d}} f^{2} d \gamma_{d}-\int_{\mathbb{R}^{d}}\left(e^{t L} f\right) d \gamma_{d} \\
& \leq\left(1-e^{-2 t}\right) \int_{\mathbb{R}^{d}}|\nabla f|^{2} d \gamma_{d}, \text { for all } t \geq 0,
\end{aligned}
$$

where $L$ is the Ornstein-Uhlenbeck operator: $L:=\Delta-x \nabla$. Recently, A.Bentaleb, S.Fahlaoui and A.Hafidi proposed in [[3], Section 2] a generalized of the inequality 3 and obtained the following inequality: for all smooth function $f$ on $\mathbb{R}^{d}$,

$$
\begin{aligned}
& \mathbb{E n t}_{\tau}^{\psi}(f):=\int_{\mathbb{R}^{d}} \psi(f) d \gamma_{d}-\int_{\mathbb{R}^{d}} \psi\left(P_{\tau} f\right) d \gamma_{d} \\
& \leq \frac{1-e^{-2 t}}{2} \int_{\mathbb{R}^{d}} \psi^{\prime \prime}|\nabla f|^{2} d \gamma_{d}
\end{aligned}
$$

where $\psi \in C^{\infty}\left(\mathbb{R}^{+}\right)$and $\psi, \frac{-1}{\psi^{\prime \prime}}$ are strictly convex, $\psi\left(0^{+}\right)=0$. Similar researches on this kind of inequalities for general probability measure generated by diffusion have been done by many authors (see, for instance, $[4,10]$ ).

The purpose of this paper is to present a family of integral inequalities on the interval $[-1,1]$ which provide interpolation between the Sobolev and Poincaré inequalities (see Theorem 1 below).

\section{Preliminaries}

In order to keep the paper reasonably self-contained, we summarize in this section the basic notion that will be used in this work.

We consider the Legendre operator $\mathrm{L}$ on the interval $I:=[-1,1]$ defined by:

$$
L:=\left(1-x^{2}\right) \frac{d^{2}}{d x^{2}}-2 x \frac{d}{d x},(x \in I),
$$

acting on acting on functions of class $C^{2}$. The Hilbert 
space $L^{2}(I, \mu)$, with respect to the probability measure $\mu(d x):=\frac{d x}{2}$. The space $L^{2}(I, \mu)$ admits orthogonal basis for the Legendre polynomials $\left(G_{k}\right)_{k \in \mathbb{N}}$ defined by the following generating series:

$$
\frac{1}{\sqrt{\left(1-2 x t+t^{2}\right)}}=\sum_{k=0}^{\infty} t^{k} G_{k} .
$$

It is Known that the Legendre polynomials are eigenvectors for the operator $-L$ :

$$
-L G_{k}=k(1+k) G_{k} \text {. }
$$

In fact, the distribution $\mu$ is symmetrizing for $L$ and the sequence $\left(-k(k+1)\right.$, $\left.\operatorname{Vect}\left(G_{k}\right)_{k \in \mathbb{N}}\right)$ forms the spectral decomposition of the minimal self-adjoint extention of this operator on $L^{2}(I, \mu)$. With the help of an integration by parts, it is easily seen that

$$
\begin{aligned}
& \forall f, g \in C^{2}(I), \\
& \int(L f) g d \mu=\int f(L g) d \mu=-\int \Gamma(f, g) d \mu,
\end{aligned}
$$

where $\Gamma$ is the positive symmetric bilinear form defined by:

$$
\Gamma(f, g)(x)=\left(1-x^{2}\right) f^{\prime}(x) g^{\prime}(x) .
$$

An important consequence of property (4) is:

$$
\forall f \in C^{2}(I), \int L f d \mu=0,
$$

which expresses the invariance of the measure $\mu$.

By means of the above mentioned properties of the operator $L$, essentially the one concerning the symmetry with respect to $\mu$, we deduce the existence of a semigroup of operator $\left(P_{t}\right)_{t \geq 0}$ generated by $L$ acting on $L^{2}(I, \mu)$ by:

$$
P_{t} G_{k}=e^{-k(k+1) t} G_{k}, \forall k \in \mathbb{N},
$$

and such that:

(1) $P_{t}$ is a contraction in all spaces $L^{p}(I, \mu)$ $(1 \leq p \leq+\infty)$;

(2) $P_{t}$ is symmetric $\int\left(P_{t} f\right) g d \mu=\int f\left(P_{t} g\right) d \mu$, $\forall f, g \in C^{2}(I)$;

(3) $P_{t}$ is positive and $P_{t} 1=1$.

According to (5), $P_{t}=e^{t L}$ and $P_{t}$ is ergodic:

$P_{t} f$ tends to $\int f d \mu \mu$-almost everywhere as $t \rightarrow+\infty$.

The commutation relation between the action of the operator $L$ and the derivation is given as:

$$
\frac{d}{d x} L=\tilde{L}\left(\frac{d}{d x}\right)-2 \frac{d}{d x}
$$

where $\tilde{L}$ is the operator associated to the family of Jacobi polynomials of second kind:

$$
\tilde{L}:=\left(1-x^{2}\right) \frac{d^{2}}{d x^{2}}-4 x \frac{d}{d x} .
$$

This commutation formula translates for the semigroup $\left(P_{t}\right)_{t \geq 0}$ by:

$$
\frac{d}{d x} P_{t}=e^{-2 t} \tilde{P}_{t}\left(\frac{d}{d x}\right)
$$

where $\tilde{P}_{t}$ designates the heat semigroup generated by $\tilde{L}$. Notice that $\tilde{P}_{t}$ is symmetric (and so invariant) with respect to the probability measure $\tilde{\mu}(d x):=\frac{3}{4}\left(1-x^{2}\right) d x$. The generator $L$ satisfies the following dissipativity formula:

$$
\int(-\tilde{L} f) g d \mu=\int\left(1-x^{2}\right) f^{\prime} g^{\prime} d \tilde{\mu},
$$

$f, g$ being sufficiently smooth on $I$. We emphasize that $\tilde{L}$ may be obtained as the projection of the Laplacian on the unit sphere $S^{4}$ and $\tilde{\mu}$ is obtained as the projection of the normalized Lebesgue measure on $S^{4}$. For $p \in[1,+\infty[$, let $D_{p}(L)$ denote the domain of the generator $L$ of $\left(P_{t}\right)_{t \geq 0}$. In virtue of density of $C^{2}(I)$ in $D_{2}(L)$, we may extend formula (4) to $D_{2}(L)$.

\section{The Main Result}

Our objective in this section is to establish a family of integral inequalities On $I=[-1,1]$ which provide interpolation between the Sobolev and Poincaré inequalities. For $p \in[1,+\infty[$, we adopt the notation

$$
L_{p}^{+}(I, \mu)=\left\{f \in L^{p}(I, \mu) ; \exists \varepsilon>0, f \geq \varepsilon\right\} .
$$

Let $\varphi: \mathbb{R}^{+} \rightarrow \mathbb{R}$ be a strictly convex function such that $\varphi(0)=0$, we define the $\varphi$-entropy functional $\mathbb{E} n t_{t}^{\psi}$ of $f \in L_{p}^{+}(I, \mu)$ by:

$$
\mathbb{E} n t_{t}^{\varphi}(f)=\int \varphi(f) d \mu-\int \varphi\left(P_{t} f\right) d \mu, p \in[0,+\infty] .
$$

The quantity $\mathbb{E} n t_{t}^{\psi}(f)$ is always nonnegative since $P_{t}$ is invariant for the probability measure $\mu$. By the ergodic property of the semigroup,

$$
\mathbb{E n t} t_{\infty}^{\varphi}(f):=\int \varphi(f) d \mu-\varphi\left(\int f d \mu\right) .
$$

When $\varphi(x)=x^{2}, \mathbb{E} n t_{\infty}^{\varphi}(f)$ coincides with the classical notion of variance,

$$
\mathbb{E n t} t_{\infty}^{\varphi}(f):=\operatorname{Var}(f)=\int f^{2} d \mu-\left(\int f d \mu\right)^{2} .
$$

When $\varphi(x)=x \log x$, 
$\mathbb{E} n t_{\infty}^{\varphi}(f):=\mathbb{E} n t(f)=\int f \log f d \mu-\int f d \mu \log \left(\int f d \mu\right)$.

In The sequel, we shall restrict ourself to the following class $C$ of real functions $\varphi \in C^{\infty}\left(\mathbb{R}^{+}\right): \varphi \in C$ mean that $\varphi(0)=0, \varphi^{\prime \prime}$ is strictly positive on $\mathbb{R}^{+}$and

$$
\frac{25}{16}\left(\varphi^{\prime \prime \prime}\right)^{2} \leq \varphi^{\prime \prime} \psi^{(I V)} \text { on } \mathbb{R}^{+} .
$$

Having in our disposal enough machinery, we are now ready to prove the following estimate of $\varphi$-entropy functional $\mathbb{E} n t_{t}^{\varphi}$ :

Theorem. 1. Let $\varphi \in C$. Then, for all function $L_{+}^{\infty}(I, \mu) \cap D_{2}(L)$ and $t \in[0,+\infty]$,

$$
\mathbb{E} n t_{t}^{\varphi}(f) \leq \frac{1}{4}\left(1-e^{-4 t}\right) \int \varphi^{\prime \prime}(f) \Gamma(f, f) d \mu .
$$

Moreover, the numeric constant at the right hand side of inequality (8) is best. To illustrate this theorem, let analyze some practical applications. The most important examples of the class $C$ in our mind are:

$$
\varphi_{p}=\frac{-x^{\frac{2}{p}}+x}{p-2} \text { for } p \in[1,34], p \neq 2
$$

and

$$
\varphi_{2}=\frac{1}{2} x \log x,
$$

which corresponds to the limiting case of $\varphi_{p}$ as $p \rightarrow+\infty$. If $\varphi=\varphi_{p}$, inequality (8), written for $t=+\infty$, describes the Sobolev inequality: for all $p \in[1,34](p \neq 2)$ and for all functions $f \in L_{\infty}^{+}(\mu, I) \cap D_{2}(L)$,

$$
\frac{\|f\|_{p}^{2}-\|f\|_{2}^{2}}{p-2} \leq \frac{1}{2} \int \Gamma(f, f) d \mu .
$$

For $\varphi=\varphi_{2}$ and $t=+\infty$ inequality (8) is exactly the Sobolev Logarithmic. Replacing f positive by $f^{2}$, we get

$$
\begin{gathered}
\mathbb{E} n t\left(f^{2}\right) \leq \frac{1}{2} \int \Gamma(f, f) d \mu, \\
\forall f \in L_{\infty}^{+}(\mu, I) \cap D_{2}(L) .
\end{gathered}
$$

Taking into account that

$$
\int \Gamma(|f|,|f|) d \mu \leq \int \Gamma(f, f) d \mu,
$$

and using the fact that set of bounded functions in $C^{2}(I)$ is dense in $D_{2}(L)$, we can extend inequalities (9) and (10) to $D_{2}(L)$. this last inequality (10) is equivalent to the hypercontractive estimate for the semigroup $\left(P_{t}\right)_{t \geq 0}$ :
Whenever $1<p<q<+\infty$ and $t>0$ satisfy $e^{4 t} \geq \frac{q-1}{p-1}$, then, for all functions $f \in L^{p}(\mu, I)$,

$$
\left\|P_{t} f\right\|_{q} \leq\|f\|_{p} .
$$

In other words, $P_{t}$ maps $L^{p}(\mu, I)$ in $L^{q}(\mu, I)(q>p)$ with norm one.

Proof. By the Fubini theorem it follows from the definition of $\mathbb{E} n t_{t}^{\varphi}(f)$ that for any $t>0$,

$$
\begin{aligned}
& \mathbb{E n t} t_{t}^{\varphi}(f)=-\int \varphi\left(P_{t} f\right)-\varphi\left(P_{0} f\right), d \mu \\
& =\int_{0}^{t} \frac{d}{d s}\left[\int \varphi\left(P_{s} f\right)-\varphi\left(P_{0} f\right), d \mu\right] d s \\
& =\int_{0}^{t}\left(\int-\left(L P_{s} f\right) \varphi^{\prime}\left(P_{s} f\right) d \mu\right) d s \\
& =\int_{0}^{t}\left(\int\left(1-x^{2}\right)\left(P_{s} f\right)^{\prime 2} \varphi^{\prime \prime}\left(P_{s} f\right) d \mu\right) d s \\
& =\int_{0}^{t} e^{-4 s}\left(\int\left(1-x^{2}\right)\left(\tilde{P}_{s} f^{\prime}\right)^{2} \varphi^{\prime \prime}\left(P_{s} f\right) d \mu\right) d s .
\end{aligned}
$$

The last tow equalities follow from the dissipativity property (4), respectively. An integration by part over the time variables yields

$$
\begin{aligned}
& \mathbb{E n} t_{t}^{\varphi}(f)=-\frac{1}{4} e^{-4 t} \int\left(1-x^{2}\right)\left(\tilde{P}_{t} f^{\prime}\right)^{2} \varphi^{\prime \prime}\left(P_{t} f\right) d \mu \\
& +\frac{1}{4} \int\left(1-x^{2}\right) f^{\prime 2} \varphi^{\prime \prime}(f) d \mu \\
& +\frac{1}{4} \int_{0}^{t} e^{-4 s} \frac{d}{d s}\left(\int\left(1-x^{2}\right)\left(\tilde{P}_{s} f^{\prime}\right)^{2} \varphi^{\prime \prime}\left(P_{s} f\right) d \mu\right) d s .
\end{aligned}
$$

Since

$$
\begin{aligned}
& \int_{0}^{t} \frac{d}{d s}\left[\int\left(1-x^{2}\right)\left(\tilde{P}_{s} f^{\prime}\right)^{2} \varphi^{\prime \prime}\left(P_{s} f\right) d \mu\right] d s \\
& =\int\left(1-x^{2}\right)\left(\tilde{P}_{t} f^{\prime}\right)^{2} \varphi^{\prime \prime}\left(P_{t} f\right) d \mu \\
& -\int\left(1-x^{2}\right) f^{\prime 2} \varphi^{\prime \prime}(f) d \mu,
\end{aligned}
$$

we get

$$
\begin{aligned}
& \mathbb{E} n t_{t}^{\varphi}(f)=\frac{1}{4}\left(1-e^{-4 t}\right) \int\left(1-x^{2}\right) f^{\prime 2} \varphi^{\prime \prime}(f) d \mu \\
& +\frac{1}{4} \int_{0}^{t}\left(e^{-4 s}-e^{-4 t}\right) \frac{d}{d s}\left[\int\left(1-x^{2}\right)\left(\tilde{P}_{s} f^{\prime}\right)^{2} \varphi^{\prime \prime}\left(P_{s} f\right) d \mu\right] d s .
\end{aligned}
$$

Now,

$$
\begin{aligned}
& e^{-4 s} \frac{d}{d s}\left[\int\left(1-x^{2}\right)\left(\tilde{P}_{s} f^{\prime}\right)^{2} \varphi^{\prime \prime}\left(P_{s} f\right) d \mu\right] d s \\
& =2 \int\left(1-x^{2}\right) \tilde{L}\left(P_{s} f\right)^{\prime} \varphi^{\prime \prime}\left(P_{s} f\right)\left(P_{s} f\right)^{\prime} d \mu \\
& +\int\left(1-x^{2}\right) L\left(P_{s} f\right)^{\prime} \varphi^{\prime \prime \prime}\left(P_{s} f\right)\left(P_{s} f\right)^{\prime 2} d \mu .
\end{aligned}
$$

Applying successively (4) and (7), the first integral in this sum is reduced to: 


$$
\begin{aligned}
& -2 \int\left(1-x^{2}\right)^{2}\left(P_{s} f\right)^{\prime \prime 2} \varphi^{\prime \prime}\left(P_{s} f\right) d \mu \\
& -2 \int\left(1-x^{2}\right)^{2}\left(P_{s} f\right)^{\prime \prime}\left(P_{s} f\right)^{\prime 2} \varphi^{\prime \prime \prime}\left(P_{s} f\right)\left(P_{s} f\right)^{\prime} d \mu,
\end{aligned}
$$

while the second integral is equal to:

$$
\begin{aligned}
& -2 \int\left(1-x^{2}\right)^{2}\left(P_{s} f\right)^{\prime 2}\left(P_{s} f\right)^{\prime \prime} \varphi^{\prime \prime \prime}\left(P_{s} f\right) d \mu \\
& -\int\left(1-x^{2}\right)^{2}\left(P_{s} f\right)^{\prime 4} \varphi^{\prime \prime \prime \prime}\left(P_{s} f\right) d \mu \\
& +2 \int x\left(1-x^{2}\right)\left(P_{s} f\right)^{\prime 3} \varphi^{\prime \prime}\left(P_{s} f\right) d \mu .
\end{aligned}
$$

Replacing $x$ by $\frac{-\tilde{L}(x)}{4}$, and invoking again the dissipativity formula (7), the last member in the preceding sum becomes:

$$
\begin{aligned}
& \frac{3}{2} \int\left(1-x^{2}\right)^{2}\left(P_{s} f\right)^{\prime 2}\left(P_{s} f\right)^{\prime \prime} \varphi^{\prime \prime \prime}\left(P_{s} f\right) d \mu \\
& +\frac{1}{2} \int\left(1-x^{2}\right)^{2}\left(P_{s} f\right)^{\prime 4} \varphi^{\prime \prime \prime \prime}\left(P_{s} f\right) d \mu .
\end{aligned}
$$

As a consequence, after reassembling the terms, we find:

$$
\begin{aligned}
& \frac{\mathbb{E} n t_{t}^{\varphi}(f)}{1-e^{-4 t}} \\
& =\frac{1}{4} \int\left(1-x^{2}\right) f^{\prime 2} \varphi^{\prime \prime}(f) d \mu \\
& -\frac{1}{4} \int_{0}^{t} \frac{1-e^{-4(t-s)}}{1-e^{-4 t}} \times\left(\int\left(1-x^{2}\right)^{2} \xi(s, f, \varphi) d \mu\right) d s
\end{aligned}
$$

with

$$
\begin{aligned}
& \xi(s, f, \varphi) \\
& =2 f_{s}^{\prime 2} \varphi^{\prime \prime}\left(f_{s}\right)+\frac{5}{2} f_{s}^{\prime 2} f_{s}^{\prime \prime} \varphi^{\prime \prime \prime}\left(f_{s}\right)+\frac{1}{2} f_{s}^{\prime 4} \varphi^{\prime \prime \prime \prime}\left(f_{s}\right) \\
& =2\left[f_{s}^{\prime \prime} \sqrt{\varphi^{\prime \prime}\left(f_{s}\right)}+\frac{5}{8} \frac{f_{s}^{\prime 2} \varphi^{\prime \prime \prime}\left(f_{s}\right)}{\sqrt{\varphi^{\prime \prime}\left(f_{s}\right)}}\right]^{2} \\
& +\frac{1}{2} \frac{f_{s}^{\prime 4}}{\varphi^{\prime \prime}\left(f_{s}\right)}\left(\varphi^{\prime \prime \prime \prime}\left(f_{s}\right) \varphi^{\prime \prime}\left(f_{s}\right)-\frac{25}{16}\left(\varphi^{\prime \prime \prime}\left(f_{s}\right)\right)^{2}\right)
\end{aligned}
$$

where we have posed $f_{s}=P_{s} f$. The of $\xi(s, f, \varphi)$ then allows us to exhibit the desired inequality (8) from (11).

It remains to show that the numeric constant $\frac{1}{4}\left(1-e^{-4 t}\right)$ at the right hand side of inequality (8) is optimal. As usual, let us consider $c \in] 0,+\infty[$ such that $\varphi^{\prime \prime}(c)>0$. If $\mathrm{f}$ is replaced by $c+\varepsilon f$ in (8), and we pass to limit as $\varepsilon$ tends to $0^{+}$, we easily recover the Poincaré inequality with best constant:

$$
\begin{aligned}
\forall t \in[0,+\infty] & , \int f^{2} d \mu-\int\left(P_{t} f\right)^{2} d \mu \\
\leq & \frac{\left(1-e^{-4 t}\right)}{2} \int \Gamma(f, f) d \mu,
\end{aligned}
$$

which completes the proof.

We close this paper by the following concluding remarks:

Of course letting $t=+\infty$, inequality (8) in Theorem 1 gives rise to:

$$
\mathbb{E}_{\infty}^{\varphi}(f) \leq \frac{1}{4} \int \Gamma(f, f) d \mu .
$$

Moreover, it's easy to observe that (8) provides a smooth nonincreasing interpolation for inequality (12):

$$
\mathbb{E} n t_{\infty}^{\varphi}(f) \leq \frac{\mathbb{E} n t_{t}^{\varphi}(f)}{1-e^{-4 t}} \leq \frac{1}{4} \int \Gamma(f, f) d \mu .
$$

By (11), we point out at once that, if $\frac{25}{16}\left(\varphi^{\prime \prime \prime}\right)^{2} \leq \varphi^{\prime \prime} \psi^{(I V)}$, the equality holds in (8) if and only if $\mathrm{f}$ is constant. In particular, inequalities (9) and (10) do not admit nonconstant extremal functions.

Corollary. 1. Let $\varphi \in C_{\infty}$. Then for all nonnegative smooth function $f[0,1] \rightarrow[0,+\infty[$,

$$
\begin{aligned}
& \mathbb{E n t}_{\mu 1}^{\varphi}(f)=\int_{0}^{1} \varphi(f(x)) x-\varphi\left(\int_{0}^{1} f(x) x\right) \\
& \leq \frac{1}{4} \int_{0}^{1} x(1-x) \psi^{\prime \prime}(f) f^{\prime 2} d x .
\end{aligned}
$$

Moreover, this inequality is optimal.

Proof. We note in the sequel by $\mu$ the uniform measure on $[0,1]$.

Let $f \in \mathcal{C}^{1}([0,1])$ positive function. We consider the function $g$ defined on the interval $[-1,1]$ by:

$$
g(x)=f\left(\frac{x+1}{2}\right)
$$

We can apply the theorem 1 to $g$ for the measure $\mu$, we obtain

$$
\begin{aligned}
& \mathbb{E n t}_{\mu_{1}}^{\varphi}(f)=\mathbb{E} n t_{\mu}^{\varphi}(g) \\
& \leq \frac{1}{4} \int\left(1-x^{2}\right) \varphi^{\prime \prime}(g) g^{\prime 2} d \mu \\
& =\frac{1}{4} \int_{0}^{1} x(1-x) \varphi^{\prime \prime}(f(x)) f^{\prime}(x)^{2} d x .
\end{aligned}
$$

\section{References}

[1] D.Bakry, M. Émery, Diffusions hypercontractives. Séminaire de probabilities de Strasbourg 19 (1985): 177-206.

[2] W. Beckner, A generalized Poincaré inequality for Gaussian measures. Proc. Am. Math. Soc. 1989; 105 (2): 397-400.

[3] A.Bentaleb,S. Fahlaoui, A.Hafidi Psi-entropy inequalities for the Ornstein-Uhlenbeck semigroup,Semigroup Forum, 2012; 85 (2): 361-368.

[4] A.Bentaleb,S.Fahlaoui, a family integral inequalities on the circle S1, Proc. Jpn. Acad., Ser. A 2010; 86: 55-59.

[5] F. Bolley, I. Gentil, Phi-entropy inequalities for diffusion semigroups. J. Math.Pures Appl. 2010; 93 (5): 449-473.

[6] J. Doulbeault, I. Gentil, and A. Jüngel, A logarithmic fourth-order parabolic equation and related logarithmic sobolev inequalitiesn Comm. Math. Sci. 2006; 4 (2): 275-290. 
[7] L.Gross,logarithmic Sobolev inequalities, Amer.J.Math. 1975; 97 (4), 1061-1083.

[8] M. Ledoux, The geometry of Markov diffusion generators. Probability theory. Ann. Fac. Sci. Toulouse Math. 2000; 6(9)(2): 305-366.
[9] E. Nelson, The free Markov field. J. Funct. Anal. 1973; 12: 211-227.

[10] F-Y. Wang, A generalisation of Poincaré and logarithmic Sobolev inequalites, Potential Anal.22(2005) no 1, 1-15. 